\title{
Sensitivity of 6 CHBT Liquid Crystal Doped with Ferroelectric or Magnetic Particles to Electric and Magnetic Fields
}

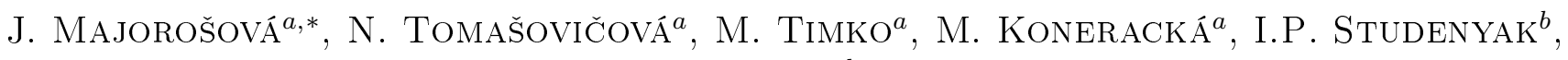 \\ O.V. KOVAlChUK ${ }^{c}$, S.O. KOVALChUK ${ }^{d}$, J. JADŻYN ${ }^{e}$, P. KOPČAnSKÝ ${ }^{a}$ \\ ${ }^{a}$ Institute of Experimental Physics, Slovak Academy of Sciences, Watsonova 47, 04001 Košice, Slovakia \\ ${ }^{b}$ Uzhorod National University 46 Pidhirna St., Uzhorod 88000, Ukraine \\ ${ }^{c}$ Institute of Physics, National Academy of Science of Ukraine,46, prospect Nauky, 03028 Kyiv, Ukraine \\ ${ }^{d}$ Taras Shevchenko National University of Kyiv 64, Volodymyrs'ka St., 01601 Kyiv, Ukraine \\ ${ }^{e}$ Institute of Molecular Physics, Polish Academy of Sciences, Smoluchowskiego 17, 60179 Poznan, Poland
}

\begin{abstract}
Introduction of ferroelectric or magnetic nanoparticles into nematic liquid crystal is a promising method for the improvement of the electro-optic or magneto-optic properties of different nematic liquid crystals by non-chemical way. This work is devoted to the study of composite systems of liquid crystal with spherical ferroelectric or magnetic particles with the aim to study their sensitivity to electric and magnetic fields. The nematic 4-(trans-4'n-hexylcyclohexyl)-isothiocyanatobenzene (6CHBT) liquid crystal was doped with SbSI ferroelectric particles or with $\mathrm{Fe}_{3} \mathrm{O}_{4}$ magnetic particles. The structural transitions in ferronematic samples were indicated by capacitance measurements in a capacitor made of ITO-coated glass electrodes in combined electric and magnetic fields. The obtained results showed the increase of the shift in critical voltage with increasing magnetic field in both kinds of samples.
\end{abstract}

DOI: $10.12693 /$ APhysPolA.126.260

PACS: $75.50 . \mathrm{Mm}, 61.30 . \mathrm{Gd}, 64.70 . \mathrm{Md}$

\section{Introduction}

Liquid crystal (LC) suspensions containing nanocolloidal particles have recently attracted great attention for many practical applications such as nanosensors, liquid crystal display industry, optical processing, biosensors, photonics and magneto-optics. The new applications are in need of new materials with exotic properties and new technologies. For example, LC materials for telecommunications usually require LCs with strong birefringence but low refractive index, adaptive LC optics needs LC materials with huge birefringence and low viscosity [1]. Ferroelectric particles have the strong effect on the optical and dielectric properties of the nematic matrix. F. Li et al. [2] observed that the increase of birefringence and dielectric anisotropy of the nematic matrix by the particles is caused by a giant dipole moment of ferroparticles that change the intermolecular interaction in the LC matrix and gives a direct contribution to the value of the effective dielectric constants of the matrix. Introduction of the particles leads to the decrease of driving voltages, increase of the reflection contrast and the steepness of the transition.

Stable colloidal suspensions of monodomain ferri- or ferromagnetic particles in nematic LC called ferronematics, attract noticeable interest because their response to an external magnetic field oversteps substantially that of pure nematics. The most essential feature of these systems is a strong orientational coupling between the

*corresponding author; e-mail: majorosova@saske.sk magnetic particles and the liquid crystal matrix. The applied magnetic field changes the orientation of the magnetic particles and due to the coupling between magnetic particles and liquid crystal molecules the director follows it. Our previous studies [3] showed that the sensitivity of ferronematics to external magnetic field depends on the concentration, type, size and shape of the magnetic particles, and on the anisotropy of the diamagnetic susceptibility of the host LC material [4].

This work is devoted to studying the sensitivity of composite systems of LC containing spherical ferroelectric or magnetic nanoparticles to electric and magnetic fields.

\section{Experiment}

Two types of samples were prepared. The thermotropic nematic 4-(trans-4'-n-hexylcyclohexyl)isothiocyanatobenzene (6CHBT) liquid crystal was doped either with spherical SbSI ferroelectric particles or $\mathrm{Fe}_{3} \mathrm{O}_{4}$ magnetic particles. Ferroelectric SbSI nanoparticles with the average grain size of $88 \mathrm{~nm}$ were obtained by ball milling the material in a stainless steel cylindrical vial with hardened steel balls. Synthesis of the magnetic nanoparticles was based on the co-precipitation of $\mathrm{Fe}^{2+}$ and $\mathrm{Fe}^{3+}$ salts by $\mathrm{NH}_{4} \mathrm{OH}$ at $60^{\circ} \mathrm{C}$ [3]. The mean diameter of magnetite particles was $10 \mathrm{~nm}$. The ferroelectric as well as the magnetic particles were of monodomain type and oleic acid was used as surfactant to avoid aggregation. The doping was done by adding these suspensions, under continuous stirring, to the liquid crystal in the isotropic phase. The volume concentration of particles was $\Phi=2 \times 10^{-3}$. The measurements of the capacitance were performed at temperature $17^{\circ} \mathrm{C}$ by a standard bridge technique 
(Andeen Hagerling). The effect of the external electric and magnetic field was studied in planar samples with thickness of $5 \mu \mathrm{m}$. The capacitor with the electrode area of approximately $1 \mathrm{~cm} \times 1 \mathrm{~cm}$ was connected to a regulated thermostatic system; the temperature was stabilized with the accuracy of $\pm 0.05^{\circ} \mathrm{C}$.

\section{Results and discussion}

The structural transitions in combined electric and magnetic fields were studied in the experimental geometry where the electric field was applied perpendicular to the capacitor electrodes and magnetic field was applied perpendicular to the electric field. We started from a planar aligned cell. First, the initial planar alignment was stabilized with the external magnetic field and than the electric field was increased, until the director of sample was reoriented to the electric field direction. The distortion manifests itself in the increase of the capacitance, that reflects the re-orientation of the nematic molecules. Figure 1 shows the dependence of the capacitance of the pure 6CHBT liquid crystal, 6CHBT doped with SbSI ferroelectric particles and $6 \mathrm{CHBT}$ doped with $\mathrm{Fe}_{3} \mathrm{O}_{4}$ magnetic particles on the electric field, without magnetic field and in magnetic field of $12 \mathrm{~T}$. These dependences show that the critical electric field is shifted to higher values after applying the magnetic field of $12 \mathrm{~T}$.

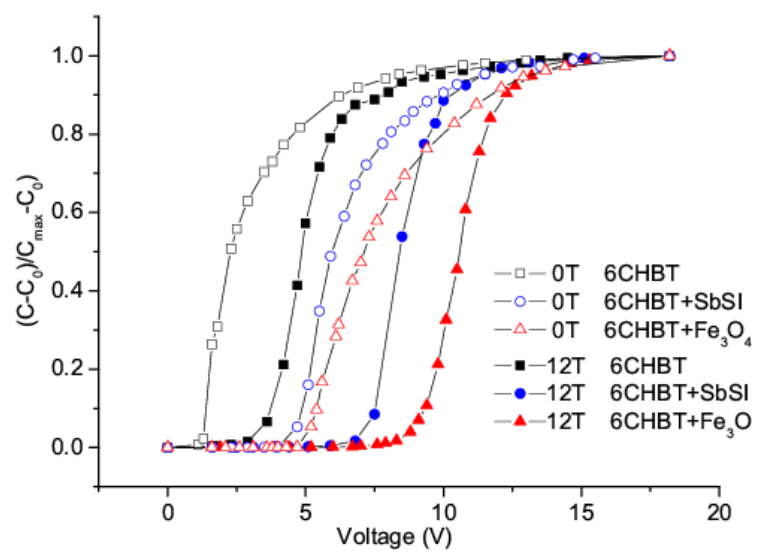

Fig. 1. Dependence of the reduced capacitance on the applied voltage without magnetic field and in magnetic field of $12 \mathrm{~T}$.

The dependences of the critical voltages on the applied magnetic field for pure 6CHBT and 6CHBT doped with SbSI or $\mathrm{Fe}_{3} \mathrm{O}_{4}$ nanoparticles are shown in Fig. 2. In the experiment the threshold of the structural transition of the pure LC and the doped LC were defined as the voltage at which the capacitance variation (indicating the distortion of the director) reached $10 \%$ of its maximum. As it is seen from Fig. 2, for pure 6CHBT, the critical voltage $\left(U_{c}\right)$ is constant for magnetic fields lower then the critical one (for 6CHBT $B_{c}=2.63 \mathrm{~T}$ ). Similarly, for 6 CHBT doped with magnetic particles, $U_{c}$ increases only slightly up to $B_{c}$, for higher magnetic field the slope of the dependence $U_{c}$ vs. $B$ increases. In our previous work [3], it was shown that the mutual orientation of the magnetic moment of the particles and the director (the unit vector which describes the preferential orientation of the nematic molecules) is parallel. In such case, the applied magnetic field hinders the reorientation of the director by electric field. On the other hand, in the case of $6 \mathrm{CHBT}$ doped with ferroelectric particles, the slope of this dependence is the same as for pure 6CHBT, but the $U_{c}$ increases with increasing magnetic field also for low values of $B$.

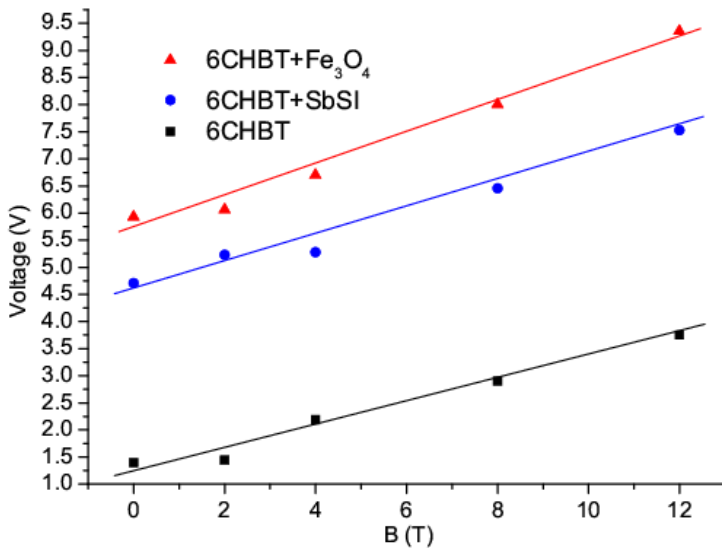

Fig. 2. Dependence of the critical voltage on the external magnetic field.

\section{Conclusions}

Our results showed that doping of 6CHBT liquid crystal with ferroelectric or magnetic particles increases the critical voltage with increasing magnetic field. These results suggest that the mutual orientation of director and electric dipole moment of particles is not parallel.

\section{Acknowledgments}

This work was supported by the Slovak Academy of Sciences grants VEGA 2/0045/13, Slovak Research and Development Agency under the contract No. APVV0171-10, Ministry of Education of Slovakia - Agency for Structural Funds of EU in frame of project No. 26110230061, 6220120021 and 6220120033, and the Grenoble High Magnetic Field Laboratory (CRETA) .

\section{References}

[1] L. Quan, Liquid crystals beyond displays: chemistry, physics, and applications, John Wiley and Sons, Inc., New Jersey 2012.

[2] F. Li, O. Buchnev, C.I. Cheon, A. Glushchenko, V. Reshetnyak, Y. Reznikov, T.J. Sluckin, J.L. West, Phys. Rev. Lett. 97, 147801 (2006).

[3] P. Kopčanský, N. Tomašovičová, M. Koneracká, V. Závišová, M. Timko, A. Džarová, A. Šprincová, N. Éber, K. Fodor-Csorba, T. Tóth-Katona, A. Vajda, J. Jadżyn, Phys. Rev. E 78, 011702 (2008).

[4] P. Kopčanský, N. Tomašovičová, M. Koneracká, M. Timko, V. Závišová, N. Éber, K. Fodor-Csorba, T. Tóth-Katona, A. Vajda, J. Jadżyn, E. Beaugnon, X. Chaud, J. Magn. Magn. Mater. 322, 3696 (2010). 